\title{
THE ALBERT ALGEBRA OF GENERIC MATRICES
}

\author{
Holger P. Petersson \\ Fachbereich Mathematik \\ FernUniversität - Gesamthochschule in Hagen \\ D - 58084 Hagen \\ Germany \\ e-mail: Holger.Petersson@FernUni-Hagen.de \\ Dieter Pumplün zur Vollendung des 65. Lebensjahres gewidmet
}

\begin{abstract}
We define the Albert algebra of generic matrices and show that its central closure is an Albert division algebra as well as a pure second Tits construction. It contains a cyclic cubic subfield iff this holds true for every Albert division algebra over any extension of the base field.
\end{abstract}

0. Introduction. Thanks to their close connection with exceptional algebraic groups of type $F_{4}$, Albert algebras, which used to be called exceptional simple Jordan algebras in the past, have attracted considerable attention over the last couple of years [10]. In particular when it comes to the classification problem, the two Tits constructions, which are known since the work of Tits (cf. Jacobson [5]) and McCrimmon [13, 14] to cover all Albert algebras over arbitrary base fields, play an important rôle. Here it is the second construction that requires considerably more care than the first. Therefore examples of pure second Tits constructions, i.e., of Albert algebras which cannot be obtained by the first, are of interest. Examples of this kind were originally supplied by Albert [2]. Later ones due to Petersson-Racine [23] and Thakur [29] have the advantage of being slightly more explicit. 
In the present paper, working over an arbitrary commutative associative ring $k$ of scalars, we define the Albert algebra of generic matrices, written as $\mathcal{A}_{k}[\mathbf{X}]$, in the spirit of Amitsur (cf. Rowen [27]) and derive some of its more elementary properties. Over fields of characteristic not 2 or 3 , a slight modification of this algebra has been studied earlier by Polikarpov [24] and Iltyakov-Shestakov [4] from the point of view of invariant theory. If $k$ is an integral domain, we show that $\mathcal{A}_{k}(\mathbf{X})$, the central closure of $\mathcal{A}_{k}[\mathbf{X}]$, is an Albert division algebra over its centroid whose quadratic trace [20] becomes anisotropic over the orthogonal complement of any separable cubic subfield; in particular, $\mathcal{A}_{k}(\mathbf{X})$ must be a pure second Tits construction. We also show that the question of Albert [2] concerning the existence of cyclic cubic subfields has an affirmative answer for all Albert division algebras if and only if this is so for $\mathcal{A}_{k}(\mathrm{X})$.

1. Preliminaries. All Jordan algebras considered in the sequel (as well as subalgebras and homomorphisms thereof) are supposed to be unital; we refer to Jacobson [6] for a systematic account of the theory.

The centroid of a Jordan algebra $J$ oder $k$ will be denoted by $\Gamma(J)$. In case this is an integral domain, with quotient field $\bar{\Gamma}(J)$, we call $J \otimes_{\Gamma(J)} \bar{\Gamma}(J)$ the central closure of $J$. If the natural map from $k$ to $\Gamma(J)$ is bijective, $J$ is said to be central. $J$ is called separable if it is finitely generated projective as $k$-module and $J \otimes \kappa(\mathfrak{p})$ is separable over the quotient field $\kappa(\mathfrak{p})$ of $k / \mathfrak{p}$ for all prime ideals $\mathfrak{p} \subset k$, unadorned tensor products always being taken over $k$. This amounts to the associated Jordan pair $(J, J)$ being separable in the sense of Loos [9].

The term " $k$-algebra" without further specification refers to unital commutative associative $k$-algebras. If $M$ is a $k$-module, we follow $[9,4$. $]$ to denote by $M_{a}$ the functor from $k$-algebras to sets which assigns to every $k$-algebra $R$ the set $M \otimes R$. Given $k$-modules $M, N$, a polynomial map from $M$ to $N$ is a natural transformation from $M_{a}$ to $N_{a}$; see Roby [26] for details and Loos $[8, \S 18]$ for a quick introduction to the subject. In particular, if $M, N$ are finite-dimensional vector spaces over an infinite field, this notion reduces to the customary one. 
By a $k$-field we mean a $k$-algebra which happens to be a field. By an extension field of $k$ we mean a $k$-field $K$ such that the natural map from $k$ to $K$ is an imbedding (forcing $k$ to be an integral domain).

2. Albert algebras over rings. We write $A^{0}$ for the neutral Albert algebra over $k$, so $A^{0}=\mathrm{H}_{3}\left(C^{0}\right)$ where $C^{0}=\operatorname{Zor}(k)$ is the neutral octonion algebra of Zorn vector matrices over $k[19,3.1]$ and $\mathrm{H}_{3}\left(C^{0}\right)$ stands for the Jordan algebra of 3-by-3 hermitian matrices with entries in $C^{0}$ and scalars down the diagonal, the latter condition being automatic if there are no 2torsions. Following Achhammer [1, 1.9], we define an Albert algebra over $k$ to be a $k$-Jordan algebra $A$ which is finitely generated projective as $k$-module and satisfies $A \otimes K \cong A^{0} \otimes K$ for all algebraically closed $k$-fields $K$. By $[1,1.10]$, or by arguing as in $[9,9$.$] , it follows that a Jordan algebra A$ over $k$ is Albert if and only if it becomes isomorphic to $A^{0}$ after a faithfully flat extension. Hence our terminology is compatible with the one of ParimalaSuresh-Thakur [17]. Also, by faithfully flat descent, all Albert algebras $A$ over $k$ arise from cubic forms with adjoint and base point $[1,1.16]: A=$ $J(V, N, \sharp, 1)$ where $V$ is a $k$-module, $N=N_{A}: V \rightarrow k$ is a cubic form (the norm of $A$ ), $\sharp: V \rightarrow V$ is a quadratic map (the adjoint of $A$ ) and $1 \in V$ is a point (the unit of $A$ ) such that, setting $T=T_{A}=-\left(D^{2} \log N\right)(1)$ (the trace of $A$ ), the relations $x^{\sharp \sharp}=N(x) x, N(1)=1, T\left(x^{\sharp}, y\right)=(D N)(x) y, 1^{\sharp}=1,1 \times$ $y=T(y) 1-y(\times$ the bilinearisation of $\sharp, T(y)=T(y, 1))$ hold in every base change of $A$. The $U$-operator of $A$ is then given by $U_{x} y=T(x, y) x-x^{\sharp} \times y$. The formula $S(x)=T\left(x^{\sharp}\right)$ defines a quadratic form $S=S_{A}: A \rightarrow k$, called the quadratic trace of $A$. Every element $x \in A$ satisfies the equation

$$
x^{3}-T(x) x^{2}+S(x) x-N(x) 1=0 .
$$

In what follows, $N, \sharp, S, T$ will be viewed as polynomial maps in the sense $\mathbf{1}$., so they act naturally (and under the same notation) on any base change of $A$. Notice also that realizing $A=J(V, N, \sharp, 1)$ as above is compatible with base change. The norm, adjoint, quadratic trace, trace of $A^{0}$ will be denoted by $N^{0}, \sharp^{0}, S^{0}, T^{0}$, respectively.

Every Albert algebra $A$ over $k$ is obviously separable. It is also central since $A^{0}$ is [14, Proposition 6] and $A$ and $A^{0}$ become isomorphic after a faithfully flat base change. 
3. Albert orders. Other sources of the literature define Albert algebras in a different way. The following result, which will also become important later on, clarifies the connections.

Theorem (McCrimmon [15, 1.27] . For a central Jordan algebra $J$ over $k$, the following statements are equivalent.

(i) There exists an extension field $K$ of $k$ and a $k$-imbedding $J \hookrightarrow A^{0} \otimes K$ whose image spans all of $A^{0} \otimes K$ over $K$.

(ii) There exists an extension field $K$ of $k$ such that $J \otimes K \cong A^{0} \otimes K$.

(iii) $J$ is generically of Albert type, i.e., $J$ is prime (forcing $k \cong \Gamma(J)$ to be an integral domain $[6,7.6 .5])$, and the central closure of $J$ is an Albert algebra over the quotient field of $k$.

A central Jordan algebra over $k$ satisfying the equivalent conditions of McCrimmon's Theorem will be called an Albert order. Indeed, if $k$ is a Dedekind domain with quotient field $K$, Albert orders over $k$ are the same as orders in the arithmetic sense of Albert algebras over $K$, see Racine [25] for details. Quite generally, Albert orders as defined here agree with Albert algebras as defined in Jacobson [6], McCrimmon [15], and Zel'manov [30]; hence, by the Zel'manov-McCrimmon structure theory [16], they are also the same as strongly prime exceptional Jordan algebras.

Corollary. A Jordan algebra over $k$ is a central separable Albert order if and only if it is an Albert algebra and $k$ is an integral domain.

Proof. Suppose first that $A$ is a central separable Albert order over $k$. By McCrimmon's Theorem, $k$ is an integral domain, and the type of the Jordan pair attached to $A[9,8$.$] defines a function from X=$ Spec $k$ to the integers which is locally constant [9, Theorem 2], hence constant since $X$ is connected (even irreducible). Therefore, as the generic fibre of $A$ is an Albert Algebra (part (iii) of McCrimmon's Theorem), so are its fibres over all points of $X$, i.e., $A$ is an Albert algebra. Conversely, let this be so and let $k$ be an integral domain. Then $A$ is central separable and its central closure agrees with its generic fibre, hence must be an Albert algebra over the quotient field of $k$. 
4. Generic matrices. Let $\boldsymbol{\xi}=\left(\xi_{i p}\right)_{1 \leq i \leq 27, p \geq 1}$ be a family of independent indeterminates and write $R=k[\boldsymbol{\xi}]$ for the corresponding polynomial ring. Once and for all we fix a $k$-basis $\left(e_{i}\right)_{1 \leq i \leq 27}$ of $A^{0}$ and consider the sequence $\mathbf{X}=\left(X_{p}\right)_{p \geq 1}$ of generic matrices

$$
X_{p}=\sum_{i=1}^{27} e_{i} \otimes \xi_{i p} \in A^{0} \otimes R \quad(p \geq 1) .
$$

We denote by $\mathcal{A}_{k}[\mathbf{X}]$ the $k$-subalgebra of $A^{0} \otimes R$ generated by the elements $X_{p}, p \geq 1$. Up to isomorphism, $\mathcal{A}_{k}[\mathbf{X}]$ does not depend on the basis chosen; it is loosely called the Albert algebra of generic matrices (although it is not an Albert algebra over $k$ in the sense of section 2), see Polikarpov [24] and Iltyakov-Shestakov [4] for slight modifications of this over fields of characteristic $\neq 2,3$ and Rowen $[27,1.3 .5]$ for the corresponding terminology in the associative case.

Observing that the element

$$
\Delta=\operatorname{det}\left(\xi_{i j}\right)_{1 \leq i, j \leq 27} \in R
$$

is not a zero divisor, we may canonically identify $R$ as a subring of the localization $R_{\Delta}$ and $A^{0} \otimes R$ as an $R$-subalgebra of $A^{0} \otimes R_{\Delta}$; also, $\Delta$ is invertible in $R_{\Delta}$. Hence, restricting (2) to indices $p \leq 27$, we conclude

$$
R_{\Delta} \mathcal{A}_{k}[\mathbf{X}]=A^{0} \otimes R_{\Delta} .
$$

This allows us to describe the centroid of $\mathcal{A}_{k}[\mathbf{X}]$ as follows.

Proposition 1. The centroid $\Gamma_{k}[\mathbf{X}]$ of $\mathcal{A}_{k}[\mathbf{X}]$ identifies canonically with the stabilizer of $\mathcal{A}_{k}[\mathbf{X}]$ under the action of $R_{\Delta}$ on $A^{0} \otimes R_{\Delta}$ by scalar multiplication:

$$
\Gamma_{k}[\mathbf{X}]=\left\{r \in R_{\Delta}: r \mathcal{A}_{k}[\mathbf{X}] \subset \mathcal{A}_{k}[\mathbf{X}]\right\} .
$$

Proof. We closely follow the argument of McCrimmon [15, 1.29 and the proof of 1.27], allowing us to skip a few details. Let $\gamma \in \Gamma_{k}[\mathbf{X}]$. Using (3), we extend $\gamma$ to a map $\gamma^{\prime}: A^{0} \otimes R_{\Delta} \longrightarrow A^{0} \otimes R_{\Delta}$ by setting

$$
\gamma^{\prime}\left(\sum r_{j} f_{j}\right)=\sum r_{j} \gamma\left(f_{j}\right)
$$


for $r_{j} \in R_{\Delta}, f_{j} \in \mathcal{A}_{k}[\mathbf{X}]$. Once we have shown that $\gamma^{\prime}$ is well defined, it is straightforward to check that it belongs to the centroid of $A^{0} \otimes R_{\Delta}$ over $R_{\Delta}$, and Proposition 1 follows. In order to prove that $(*)$ makes sense, we choose $r_{j}, f_{j}$ as above satisfying $\sum r_{j} f_{j}=0$ to establish $\sum r_{j} \gamma\left(f_{j}\right)$ as an element of

$\operatorname{Ker}\left(A^{0} \otimes R_{\Delta}\right)=\left\{x \in A^{0} \otimes R_{\Delta}: U_{x}=U_{x, y}=0\right.$ for all $\left.y \in A^{0} \otimes R_{\Delta}\right\}$,

which, as noted in $[6,5.4]$, is zero for all standard Jordan matrix algebras.

5. Polynomial identities. Let $\mathcal{J}_{k}[\mathbf{Y}]$ be the free Jordan algebra over $k$ in a sequence $\mathbf{Y}=\left(Y_{p}\right)_{p \geq 1}$ of independent generators $Y_{1}, Y_{2}, \ldots$ Given any Jordan algebra $J$ over an arbitrary $k$-algebra and any sequence $\mathbf{u}=\left(u_{p}\right)_{p \geq 1}$ in $J$, the evaluation

$$
\varepsilon_{\mathbf{u}}: \mathcal{J}_{k}[\mathbf{Y}] \longrightarrow J, f \longmapsto f(\mathbf{u})=\varepsilon_{\mathbf{u}}(f),
$$

is the unique $k$-homomorphism sending $Y_{p}$ to $u_{p}$ for $p \geq 1$. As in the associative case $[27,1.1 .12], f \in \mathcal{J}_{k}[\mathbf{Y}]$ is said to be an identity for $J$ (as a $k$-algebra) if $f(\mathbf{u})=0$ for all sequences $\mathbf{u}$ in $J$ as above. Notice that each $f \in \mathcal{J}_{k}[\mathbf{Y}]$ depends on a finite number of $Y$ 's only: $f=f\left(Y_{1}, \ldots, Y_{n}\right), n \in \mathbb{N}$ varying with $f$, and so may be regarded as a polynomial map $J^{n} \longrightarrow J$ via evaluation. In particular, for $f \neq 0$ and $J$ finite-dimensional over an infinite $k$-field, we conclude $f(\mathbf{u}) \neq 0$ on a Zariski-dense subset of $J^{n}$. Notice also that the entire set-up especially applies to $J=\mathcal{A}_{k}[\mathbf{X}], \mathbf{u}=\mathbf{X}$. The following result and its corollaries have wellknown associative analogues whose proofs we will follow closely, cf. Rowen [27, 1.3.6 - 1.3.11] for details.

Proposition 2. Notations being as in 4., 5., let $k^{\prime}$ be a $k$-algebra and $A$ an Albert algebra over $k^{\prime}$. Given a sequence $\mathbf{u}=\left(u_{p}\right)_{p \geq 1}$ of elements in $A$, there exists a unique $k$-homomorphism $\varepsilon_{\mathbf{u}}^{*}: \mathcal{A}_{k}[\mathbf{X}] \longrightarrow A$ sending $X_{p}$ to $u_{p}$ for all $p \geq 1$. More specifically, given any faithfully flat $k^{\prime}$-algebra $k^{\prime \prime}$ and any isomorphism $\Phi: A^{0} \otimes k^{\prime \prime} \stackrel{\sim}{\longrightarrow} A \otimes_{k^{\prime}} k^{\prime \prime}$ over $k^{\prime \prime}$ (the existence of $k^{\prime \prime}$, $\Phi$ being assured by 2.), there exists a unique $k$-homomorphism $\alpha_{\mathbf{u}}: R \longrightarrow k^{\prime \prime}$ such that $\varepsilon_{\mathbf{u}}^{*}$ is induced from $\Phi \circ\left(\mathbf{1}_{A^{\circ}} \otimes \alpha_{\mathbf{u}}\right)$ via restriction. Finally, the relations

$$
N_{A}\left(\varepsilon_{\mathbf{u}}^{*}(x)\right)=\alpha_{\mathbf{u}}\left(N^{0}(x)\right),
$$




$$
\begin{aligned}
T_{A}\left(\varepsilon_{\mathbf{u}}^{*}(x), \varepsilon_{\mathbf{u}}^{*}(y)\right) & =\alpha_{\mathbf{u}}\left(T^{0}(x, y)\right), \\
S_{A}\left(\varepsilon_{\mathbf{u}}^{*}(x)\right) & =\alpha_{\mathbf{u}}\left(S^{0}(x)\right)
\end{aligned}
$$

hold for all $x, y \in \mathcal{A}_{k}[\mathbf{X}]$.

Proof. Uniqueness of $\varepsilon_{\mathbf{u}}^{*}$ is obvious. To prove its existence, we write $\Phi^{-1}\left(u_{p}\right) \in$ $A^{0} \otimes k^{\prime \prime}$ in the form

$$
\Phi^{-1}\left(u_{p}\right)=\sum_{i=1}^{27} e_{i} \otimes b_{i p}, b_{i p} \in k^{\prime \prime} \text { for } 1 \leq i \leq 27, p \geq 1,
$$

and consider the unique $k$-homomorphism $\alpha_{\mathbf{u}}: R \longrightarrow k^{\prime \prime}$ sending $\xi_{i p}$ to $b_{i p}(1 \leq i \leq 27, p \geq 1)$. Then $\Phi \circ\left(\mathbf{1}_{A^{0}} \otimes \alpha_{\mathbf{u}}\right)$ maps each $X_{p}$ to $u_{p}$ (a property which determines $\alpha_{\mathbf{u}}$ uniquely) and hence yields $\varepsilon_{\mathbf{u}}^{*}$ as claimed. The three formulae at the very end follow from the fact that polynomial maps are compatible with base change.

Corollary 1. For $f \in \mathcal{J}_{k}[\mathbf{Y}]$ the following statements are equivalent.

(i) $f$ is an identity for $A^{0} \otimes R$.

(ii) $f$ is an identity for $\mathcal{A}_{k}[\mathbf{X}]$.

(iii) $f(\mathbf{X})=0$.

Proof. Since $\mathcal{A}_{k}[\mathbf{X}]$ is a $k$-subalgebra of $A^{0} \otimes R$, (i) implies (ii). The implication (ii) $\Longrightarrow$ (iii) being obvious, it remains to prove (iii) $\Longrightarrow$ (i). Assume (iii) and let $\mathbf{u}=\left(u_{p}\right)_{p \geq 1}$, be a sequence in $A^{0} \otimes R$. Then Proposition 2 yields a unique $k$-homomorphism $\varepsilon_{\mathbf{u}}^{*}: \mathcal{A}_{k}[\mathbf{X}] \longrightarrow A^{0} \otimes R$ sending $X_{p}$ to $u_{p}$ for $p \geq 1$. It follows $f(\mathbf{u})=\varepsilon_{\mathbf{u}}^{*}(f(\mathbf{X}))=0$.

Given Jordan algebras $J_{1}, J_{2}$ ober $k$, we write $J_{1} \leq_{k} J_{2}$ if every identity valid in $J_{2}$ is valid in $J_{1}$ as well, see Rowen $[27$, p. 5] for the corresponding terminology in the associative setting.

Corollary 2. $\mathcal{A}_{k}[\mathbf{X}]$ is the free object on $\mathbf{X}$ in the category of $k$-Jordan algebras $\leq_{k} A^{0} \otimes R$.

Proof. We have $\mathcal{A}_{k}[\mathbf{X}] \leq_{k} A^{0} \otimes R$ by Corollary 1. Let $J$ be any $k$-Jordan algebra $\leq_{k} A^{0} \otimes R$ and $\mathbf{u}=\left(u_{p}\right)_{p \geq 1}$ a sequence in $J$. Every element $f \in$ ker $\varepsilon_{\mathbf{X}} \subset \mathcal{J}_{k}[\mathbf{Y}]$ is an identity for $A^{0} \otimes R$ (Corollary 1 ), hence for $J$, and we 
conclude $f(\mathbf{u})=0$. Hence ker $\varepsilon_{\mathbf{X}} \subset \operatorname{ker} \varepsilon_{\mathbf{u}}$, so $\varepsilon_{\mathbf{u}}$ factors uniquely through $\mathcal{A}_{k}[\mathbf{X}]$.

Remark 1. Assuming that $k$ is a field, $k^{\prime}$ is a field extension $F / k$ and $k^{\prime \prime}$ stands for the algebraic closure $\bar{F}$ of $F$, Proposition 2 can be combined with the following observation. Let $m \in \mathbb{N}, V$ be a finite-dimensional vector space over $k$ and $P: A^{0 m} \longrightarrow V$ a polynomial map. Identifying $A^{0} \otimes \bar{F}=A \otimes_{F} \bar{F}=\bar{A}$ by means of $\Phi, P$ determines a polynomial map $P: \bar{A}^{m} \longrightarrow V \otimes \bar{F}$ in the classical sense, forcing

$$
\left\{x \in A^{m}: P(x) \neq 0\right\}
$$

to be open dense in $A^{m}$ relative to the Zariski topology over $F$ if $P$ is nonzero and $F$ is infinite.

Remark 2. E. Neher has pointed out that, with proper adjustments, the results obtained up to now remain valid under more general circumstances, replacing the neutral Albert algebra $A^{0}$ by any central separable Jordan algebra over $k$ which is free as a $k$-module.

6. The central closure. For the rest of the paper we assume that $k$ is an integral domain. This property carries over to the centroid of $\mathcal{A}_{k}[\mathbf{X}]$ (Proposition 1), allowing us to pass to the central closure

$$
\mathcal{A}_{k}(\mathbf{X})=\mathcal{A}_{k}[\mathbf{X}] \otimes_{\Gamma_{k}[\mathbf{X}]} \Gamma_{k}(\mathbf{X}) \subset A^{0} \otimes K
$$

$\Gamma_{k}(\mathbf{X}), K$ being the quotient field of $\Gamma_{k}[\mathbf{X}], R$, respectively. Since $\mathcal{A}_{k}[\mathbf{X}]$ by (3) spans all of $A^{0} \otimes K$ as a vector space over $K$, we conclude from McCrimmon's Theorem (3.) that $\mathcal{A}_{k}(\mathbf{X})$ is an Albert algebra over $\Gamma_{k}(\mathbf{X})$.

Remark 3. (i) The last conclusion, a slightly different version of which has already been stated by Iltyakov-Shestakov [4, p. 840] over fields of characteristic not 2 or 3 , in full generality is really the sticky point of the paper. Analyzing the proof of McCrimmon's Theorem shows that this conclusion rests on the existence of central multiplication identities for Albert algebras $[15,(1.20), 1.23,1.26]$ and is therefore unlikely, presently at least, to extend intrinsically to arbitrary central separable Jordan algebras as in Remark 2. 
(ii) The natural imbedding $\mathcal{A}_{k}[\mathbf{X}] \hookrightarrow A^{0} \otimes K$ induces a $K$-homomorphism $\mathcal{A}_{k}(\mathbf{X}) \otimes_{\Gamma_{k}(\mathbf{X})} K \longrightarrow A^{0} \otimes K$ which is surjective by (3) and injective by outer simplicity [14, Theorem 5]. We identify $\mathcal{A}_{k}(\mathbf{X}) \otimes_{\Gamma_{k}(\mathbf{X})} K=A^{0} \otimes K$ accordingly. Then the norm, adjoint, quadratic trace, trace of $A^{0}$ over $k$ yield the corresponding objects of $\mathcal{A}_{k}(\mathbf{X})$ over $\Gamma_{k}(\mathbf{X})$ via restriction from $A^{0} \otimes K$.

7. Main results. We are now prepared to state the main results of the paper. After having established a few indispensable prerequisites, proofs will be given in section $\mathbf{1 0}$. below.

Theorem 1. Notations being as in $\mathbf{6 .}, \mathcal{A}_{k}(\mathbf{X})$ is an Albert division algebra over $\Gamma_{k}(\mathbf{X})$ and a pure second Tits construction.

Using the formula

$$
T(x \times y)=T(x) T(y)-T(x, y),
$$

valid in every Jordan algebra $J$ of degree 3 [13, p. 497], it follows that the orthogonal complement of an étale (= separable commutative associative) subalgebra of degree 3 in $J$ relative to the trace agrees with the one relative to the quadratic trace. With this in mind, we obtain

Theorem 2. The quadratic trace of $\mathcal{A}_{k}(\mathbf{X})$ over $\Gamma_{k}(\mathbf{X})$ is anisotropic on the orthogonal complement of any separable cubic subfield.

Remark 4. By contrast, the quadratic trace is always isotropic on the separable cubic subfield itself $[22,3.3]$.

Theorem 3. If $\mathcal{A}_{k}(\mathbf{X})$ over $\Gamma_{k}(\mathbf{X})$ admits a cyclic cubic subfield, so does every Albert division algebra over any extension field of $k$.

8. Extension fields. In this section we assume that $k$ is a field to establish the existence of certain division algebras and anisotropic quadratic forms over suitable extension fields of $k$. Our results are routine but indispensable. 
Lemma 1. There exist Albert division algebras over suitable extension fields of $k$.

Proof. By passing to $k(t), t$ an indeterminate, and considering the polynomial $T^{3}+t T+t$ over $k(t)$, we may assume that $k$ admits a separable cubic extension $E / k$. By passing to a suitable quadratic extension if necessary, we may assume that $E / k$ is cyclic. By passing to $k(t)$ again, we may assume that the norm $N_{E / k}$ is not surjective [5, IX, 12 Lemma 1, p. 417], allowing us to construct a cyclic division algebra $D$ of degree 3 over $k$ [7, Exercise 8.5.3]. Repeating the preceding argument, we may assume that the generic norm of $D$ is not surjective. But then the first Tits construction [13, Theorem 6] yields an Albert division algebra over $k$.

There is another result along similar lines whose proof turns out to be even easier and will therefore be omitted.

Lemma 2. There exist octonion division algebras over suitable extension fields of $k$.

Lemma 3. Let $m$ be a positive integer and $\left(q_{1}, \ldots, q_{m}\right)$ (resp. $T=$ $\left(T_{1}, \ldots, T_{m}\right)$ ) be a family of anisotropic quadratic forms (resp. of independent indeterminates) over $k$. Then the quadratic form

$$
T_{1} q_{1} \perp \ldots \perp T_{m} q_{m}
$$

is anisotropic over $k(T)$.

Proof. By induction we reduce to the case $m=2$ and must show with a single indeterminate $t$ that $q_{1}+t q_{2}$ is anisotropic over $k(t)$. Writing $V_{i}(i=1,2)$ for the vector space over $k$ on which $q_{i}$ is defined and $x_{i} \mapsto x_{i}(0)$ for the map $V_{i} \otimes k[t] \rightarrow V_{i}$ given by polynomial evaluation at 0 , we argue indirectly to find elements $x_{i} \in V_{i} \otimes k[t]$ satisfying

$$
q_{1}\left(x_{1}\right)+t q_{2}\left(x_{2}\right)=0,\left(x_{1}(0), x_{2}(0)\right) \neq(0,0) .
$$

Setting $t=0$ yields $x_{1}(0)=0$, hence $x_{1}=t y_{1}$ for some $y_{1} \in V_{1} \otimes k[t]$. Substituting, dividing by $t$ and setting $t=0$ again yields $x_{2}(0)=0$ as well, a contradiction. 
Remark 4. For char $k \neq 2$ the case $m=2$ above can also be understood by passing to the field $k((t))$ of formal Laurent series over $k$ and by considering residue class forms in the sense of Springer, cf. Scharlau [28, 6.2.6].

Lemma 4. Let $\left(q_{1}, q_{2}, q_{3}\right)$ (resp. $\left.T=\left(T_{1}, T_{2}, T_{3}\right)\right)$ be a triple of anisotropic quadratic forms (resp. of independent indeterminates) over $k$. Then the quadratic form

$$
<-1>\perp\left(T_{2} T_{3} q_{1}\right) \perp\left(T_{3} T_{1} q_{2}\right) \perp\left(T_{1} T_{2} q_{3}\right)
$$

is anisotropic over $k(T)$.

Proof. The relations

$$
\frac{\left(T_{i} T_{i+1}\right)\left(T_{i+1} T_{i+2}\right)}{T_{i+2} T_{i}}=T_{i+1}^{2}
$$

show that $T_{2} T_{3}, T_{3} T_{1}, T_{1} T_{2}$ are algebraically independent over $k$. Hence Lemma 3 applies with $q_{0}=\langle-1\rangle$ and an extra variable $T_{0}$ to complete the proof.

9. Reduced Albert algebras. Assume that $k$ is a field and let $A$ be a reduced Albert algebra over $k$, so $A$ may be coordinatized as $A=\mathrm{H}_{3}(C, g)$ where $C$ is an octonion algebra, $g \in \mathrm{GL}_{3}(k)$ is a diagonal matrix and $\mathrm{H}_{3}(C, g)$ stands for the Jordan algebra of 3-by-3 $g$-hermitian matrices $\left(x=g^{-1 t} \bar{x} g\right)$ having entries in $C$ and scalars down the diagonal. More specifically, we denote by $n=n_{C}$ the norm of $C$, set $g=\operatorname{diag}\left(g_{1}, g_{2}, g_{3}\right)$ and employ the customary notation for the hermitian matrix units [13, p. 502], allowing us to write $x, y \in A$ as

$$
x=\sum a_{i} e_{i}+\sum x_{i}[j l], y=\sum b_{i} e_{i}+\sum y_{i}[j l],
$$

$a_{i}, b_{i} \in k, x_{i}, y_{i} \in C(1 \leq i \leq 3)$, unspecified sums always being extended over the cyclic permutations $(i j l)$ of (123). Then the trace and quadratic trace of $A$ are given by

$$
\begin{aligned}
T(x, y) & =\sum a_{i} b_{i}+\sum g_{j} g_{l} n\left(x_{i}, y_{i}\right), \\
S(x) & =\sum a_{j} a_{l}-\sum g_{j} g_{l} n\left(x_{i}\right) .
\end{aligned}
$$


The 3-by-3 diagonal matrices over $k$ comprise a split étale subalgebra $E^{0}$ of $A$, and restricting $S$ to the orthogonal complement of $E^{0}$ by $(3)$, (4) yields a quadratic form

$$
Q=Q_{A} \cong-\left(\left(g_{2} g_{3} n\right) \perp\left(g_{3} g_{1} n\right) \perp\left(g_{1} g_{2} n\right)\right),
$$

which up to isometry is independent of the coordinatization chosen [23, 2.2]. Following Faulkner [3, Lemma 1.5], $A_{0}\left(e_{1}\right)$, the Peirce-zero-subalgebra of $A$ relative to $e_{1}$, is given by

$$
A_{0}\left(e_{1}\right)=k e_{2}+k e_{3}+C[23]
$$

and, in fact, agrees with the Jordan algebra of the quadratic form $S$ on this space with base point $e_{2}+e_{3}$.

Proposition 3. Notations being as above, suppose the quadratic form

$$
<-1>\perp Q_{A}
$$

is anisotropic. Then so is $S_{A}$ on the orthogonal complement of any étale rank-3-subalgebra of $A$.

Proof. Let $E \subset A$ be an étale subalgebra of rank 3. By passing to the base change $A \otimes E$ if necessary, which is justified by Springer's Theorem (cf. [22, 2.3] for a formulation in arbitrary characteristic), we may assume $E=k \oplus E^{\prime}$ where $E^{\prime}$ is étale of rank 2 over $k$. Then $E^{\prime}$ is generated by an element $z$ solving the minimum equation $z^{2}-z+\theta 1_{E^{\prime}}=0$ for some $\theta \in k$. In view of (8), we can find a coordinatization $A=\mathrm{H}_{3}(C, g)$ as above such that

$$
z=c_{2} e_{2}+c_{3} e_{3}+z_{1}[23]
$$

where $c_{2}, c_{3} \in k, z_{1} \in C$ satisfy the relations $c_{2}+c_{3}=1, c_{2} c_{3}-g_{2} g_{3} n\left(z_{1}\right)=\theta$ and $E$ is spanned as a vector space by $e_{1}, e_{2}+e_{3}, z$. Combining (5) with (9) we conclude that $E^{\perp}$ consists of all elements

$$
x=a\left(e_{2}-e_{3}\right)+\sum x_{i}[j l]
$$

satisfying $a \in k, x_{i} \in C,\left(c_{3}-c_{2}\right) a=g_{2} g_{3} n\left(z_{1}, x_{1}\right)$. Evaluating $S=S_{A}$ at such an element yields

$$
S(x)=-a^{2}-\sum g_{j} g_{l} n\left(x_{i}\right)
$$

by (6), and a comparison with (7) completes the proof. 
10. Proofs of the main results. We have assembled now all the necessary machinery to carry out the proofs of Theorems 1,2,3 of $\mathbf{7}$. above.

Proof of Theorem 1, part I. We know that $\mathcal{A}_{k}(\mathbf{X})$ is an Albert-Algebra over $\Gamma_{k}(\mathbf{X})$ (6.).To show that it is a division algebra, we pick a nonzero element $x \in \mathcal{A}_{k}(\mathbf{X})$ and must prove $N^{0}(x) \neq 0$ (Remark 3 (ii)). Since there is no harm in assuming $x \in \mathcal{A}_{k}[\mathbf{X}]$, we have $x=f(\mathbf{X})$ for some nonzero $f \in \mathcal{J}_{k}[\mathbf{Y}]$. Applying Lemma 1, we find a field extension $F / k$ and an Albert division algebra $A$ over $F$. Since finite Jordan division rings canonically identify with their centroids (McCrimmon [12], Petersson [18]), $F$ must be infinite, so there exists a sequence $\mathbf{u}=\left(u_{p}\right)_{p \geq 1}$ in $A$ such that $f(\mathbf{u}) \neq 0$, forcing $N_{A}(f(\mathbf{u})) \neq 0$. But then $N^{0}(x) \neq 0$ by Proposition 2 .

Proof of Theorem 2. Combining Lemmata 2,4 with (7) and Proposition 3, we find a field extension $F / k$ and a reduced Albert algebra $A$ over $F$ whose quadratic trace is anisotropic on the orthogonal complement of any étale rank-3-subalgebra of $A$. Now let $E \subset \mathcal{A}_{k}(\mathbf{X})$ be a separable cubic subfield. Picking a generator $x \in \mathcal{A}_{k}[\mathbf{X}]$ of $E / \Gamma_{k}(\mathbf{X})$, we conclude $x=f(\mathbf{X})$ for some $f \in \mathcal{J}_{k}[\mathbf{Y}]$ and

$$
\operatorname{det}\left(T^{0}\left(x^{i}, x^{j}\right)\right)_{0 \leq i, j \leq 2} \neq 0 .
$$

Hence Remark 1 yields a sequence $\mathbf{u}$ in $A$ such that $u=f(\mathbf{u}) \in A$ satisfies

$$
\operatorname{det}\left(T_{A}\left(u^{i}, u^{j}\right)\right)_{0 \leq i, j \leq 2} \neq 0
$$

in other words: $u$ generates an étale subalgebra $F[u]$ of rank 3 in $A$. Suppose now that $S^{0}$ is isotropic on $E^{\perp}$. Then there exists a nonzero $y=g(\mathbf{X}) \in$ $\mathcal{A}_{k}[\mathbf{X}], g \in \mathcal{J}_{k}[\mathbf{Y}]$, satisfying the relations $S^{0}(y)=T^{0}\left(x^{i}, y\right)=0$ for $i=0,1,2$. Hence Remark 1 yields a sequence $\mathbf{v}$ in $A$ having $v=g(\mathbf{v}) \neq 0$, and by Proposition 2, $v \in F[u]^{\perp}$ is isotropic relative to $S_{A}$, a contradiction.

Proof of Theorem 1, part II. Since an Albert division algebra which is a first Tits construction splits under a suitable cubic extension [20, Corollary 4.2], its quadratic trace for char $k \neq 2$ (resp. the restriction thereof to the elements of trace zero for char $k=2$ ) has maximal Witt index, by Springer's Theorem and $[23,2.2]$, forcing it to become isotropic on the orthogonal complement of any separable cubic subfield. Hence, by Theorem $2, \mathcal{A}_{k}(\mathbf{X})$ must be a pure second Tits construction. 
Proof of Theorem 3. A cubic field extension is cyclic if and only if it contains distinct elements having the same minimum polynomial. Assuming $E \subset$ $\mathcal{A}_{k}(\mathbf{X})$ to be a cyclic cubic subfield, we therefore find $x=f(\mathbf{X}) \neq y=g(\mathbf{X})$ in $\mathcal{A}_{k}[\mathbf{X}], f, g \in \mathcal{J}_{k}[\mathbf{Y}]$, satisfying, because of (1),

$$
\begin{gathered}
\operatorname{det}\left(T^{0}\left(x^{i}, x^{j}\right)\right)_{0 \leq i, j \leq 2} \neq 0, \\
N^{0}(x)=N^{0}(y), S^{0}(x)=S^{0}(y), T^{0}(x)=T^{0}(y), \\
y=\varphi_{0} 1+\varphi_{1} x+\varphi_{2} x^{2}
\end{gathered}
$$

for some $\varphi_{0}, \varphi_{1}, \varphi_{2} \in \Gamma_{k}(\mathbf{X})$. Let $A$ be an Albert division algebra over an extension field $F / k$. Then Proposition 2 and Remark 1 yield a sequence $\mathbf{u}$ in $A$ such that $u=f(\mathbf{u}) \neq g(\mathbf{u})=v$ and

$$
\begin{gathered}
\operatorname{det}\left(T_{A}\left(u^{i}, u^{j}\right)\right)_{0 \leq i, j \leq 2} \neq 0, \\
N_{A}(u)=N_{A}(v), S_{A}(u)=S_{A}(v), T_{A}(u)=T_{A}(v), \\
v=b_{0} 1+b_{1} u+b_{2} u^{2}
\end{gathered}
$$

with coefficients $b_{0}, b_{1}, b_{2}$ in the algebraic closure $\bar{F}$ of $F$. Thus $F[u] \subset A$ is a cubic subfield, and $v \in \bar{F}[u] \cap A=F[u]$ by (1) has the same minimum polynomial as $u$. Hence $F[u]$ is cyclic over $F$.

\section{ACKNOWLEDGEMENTS}

The author is indebted to O. Loos, M. L. Racine, C. Scheiderer and, particularly, to E. Neher for useful advice.

\section{REFERENCES}

1. Achhammer, G. "Albert-Algebren über lokal geringten Räumen". Dissertation. Hagen 1995.

2. Albert, A. A. On exceptional Jordan division algebras. Pacific J. Math. 16 (2) (1965), 377-404. 
3. Faulkner, J. R. "Octonion planes defined by quadratic Jordan algebras." Mem. Amer. Math. Soc. 104 (1970).

4. Iltyakov, A. I. and I. P. Shestakov. On invariants of $F_{4}$ and the center of the Albert Algebra. J. Algebra 179 (1996), 838 - 851.

5. Jacobson, N. "Structure and representations of Jordan algebras." Amer. Math. Soc. Coll. Publ. 39, Providence, RI, 1968.

6. - "Structure theory of Jordan algebras". Fayetteville, AR, USA: University of Arkansas Lecture Notes, 1981.

7. - "Basic Algebra II." Second Edition. New York: W. H. Freeman, 1989.

8. Loos, O. "Jordan pairs". Lecture Notes in Mathematics 460. Berlin Heidelberg New York: Springer, 1975.

9. $\quad$ - Separable Jordan pairs over commutative rings. Math. Ann. 233 (1978), 137-144.

10. Knus, M.-A., A. S. Merkurjev, M. Rost, J.-P. Tignol "The book of involutions." Amer. Math. Soc. Coll. Publ. 44, Providence, RI, 1998.

11. Lang, S. "Algebra." Reading, MS: Addison-Wesley, 1965.

12. McCrimmon, K. A note on finite division rings. Proc. Amer. Math. Soc. 23 (1969), 598-600.

13. - The Freudenthal-Springer-Tits constructions of exceptional Jordan algebras. Trans. Amer. Math. Soc. 139 (1969), 495-510.

14. - The Freudenthal-Springer-Tits constructions revisited. Trans. Amer. Math. Soc. 148 (1970), 293-314.

15. - Zelmanov's prime theorem for quadratic Jordan algebras. J. Algebra 76 (2) (1982), 297-326.

16. McCrimmon, K. and E. I. Zel'manov. The structure of strongly prime quadratic Jordan algebras. Advances Math. 69 (2) (1988), 133222 . 
17. Parimala, R., V. Suresh and M. L. Thakur. Jordan algebras and $F_{4}$ bundles over the affine plane. J. Algebra 198 (1997), 582-607.

18. Petersson, H. P. Jordan-Divisionsalgebren und Bewertungen. Math. Ann. 202 (1973), 215-243.

19. - Composition algebras over algebraic curves of genus zero. Trans.Amer. Math. Soc. 337 (1993), 473-493.

20. Petersson, H. P. and M. L. Racine Springer forms and the first Tits construction of exceptional simple Jordan algebras. Manuscripta Math. 45 (1984), 249-272.

21. - Pure and generic first Tits constructions of exceptional Jordan division algebras. Algebras Groups Geom. 3 (1986), 386-398.

22. - On the invariants mod 2 of Albert algebras. J. Algebra 174 (1995), 1049-1072.

23. - Reduced models of Albert algebras. Math. Z. 222 (1996), 367-385.

24. Polikarpov, S. V. Free affine Albert algebras. Sibirsk. Math. Zh. 32 (6) (1991), $131-141$.

25. Racine M. L. "The arithmetics of quadratic Jordan algebras". Mem. Amer. Math. Soc. 136, Providence, RI, 1973.

26. Roby, N. Lois polynômes et lois formelles en théorie des modules. Ann. Sci. Ecole Norm. Sup. $3^{e}$ ser. t. 80 (1963), 213-348.

27. Rowen, H. L. "Polynomial identities in ring theory". New York London Toronto Sydney San Francisco: Academic Press, 1980.

28. Scharlau, W. "Quadratic and hermitian forms." Grundlehren der mathematischen Wissenschaften 270. Berlin Heidelberg: Springer 1985.

29. Thakur, M. L. Isotopy and Invariants of Albert Algebras. To appear.

30. Zel'manov, E. I. On prime Jordan rings. Algebra i Logika 18 (1979), $162-175$. 